Introduction Since 2015, mechanical thrombectomy is the standard treatment for emergent large vessel occlusion ischemic stroke. The Blood and Clot Thrombectomy Registry and Collaboration (BACTRAC) protocol (clinicaltrials.gov NCT03153683) utilizes thrombectomy to isolate intracranial (i. e. distal to thrombus) arterial blood, systemic arterial blood, and the thrombus itself. We set out to investigate how the protein expression of a patient's intracranial blood during ischemic stroke compares to the protein expression of their systemic arterial blood in order to better understand and treat stroke.

Methods Intracranial and systemic plasma samples from $n=25$ subjects were randomized and sent for cardiometabolic and inflammatory proteomic analyses at Olink Proteomics (Olink Proteomics, Boston, MA). Olink provides a Normalized Protein eXpression (NPX), a unit that is in $\log 2$ scale which allows for individual protein analysis across a sample set. The intracranial versus systemic fold change in NPX was calculated by subtracting the systemic NPX $\left(\mathrm{NPX}_{\mathrm{s}}\right)$ from the intracranial NPX $\left(\mathrm{NPX}_{\mathrm{i}}\right)$. In order to determine which proteins had the most significant changes, a series of 184 paired t-tests were performed, one for each of the 92 cardiometabolic and 92 inflammatory proteins. Data analysis was performed using SAS software version 9.4 (SAS Institute Inc., Cary, N.C.).

Results 83 proteins with significantly different expression levels between intracranial and systemic blood were identified and all 83 exhibited lower expression in the intracranial blood. For the cardiometabolic panel, the 5 most significant fold changes were: prolyl endopeptidase (FAP) at $-0.26(\mathrm{p}<0.0001)$, phospholipid transfer protein (PLTP) at $-0.26(\mathrm{p}=0.0005)$, uromodulin (UMOD) at $-0.14(\mathrm{p}=0.002)$, fetuin-B (FETUB) at -0.31 $(p=0.002)$, and ficolin-2 (FCN2) at $-0.46(p=0.005)$. For the inflammatory panel, the 5 most significant fold changes were: C-C motif chemokine 19 (CCL19) at -0.51 ( $p<0.0001)$, C-C motif chemokine $20($ CCL20) at $-0.40(\mathrm{p}<0.0001)$, fibroblast growth factor 21 (FGF21) at $-0.37(\mathrm{p}=0.0002)$, transforming growth factor alpha (TGF-a) at $-0.28 \quad(\mathrm{p}=0.0002)$, and C-C motif chemokine (CCL23) at $-0.43(p=0.0003)$.

Discussion We have, for the first time, evaluated proteomic changes in the intravascular space of a cerebral infarct in-progress in human subjects. Interestingly, for all significant proteins, expression levels were lower in the intracranial blood compared to systemic. The most significantly changed proteins may represent specific responses occurring at the time of ischemia. For example, changes in proteins such as SOD1 and FGF21 may play a neuroprotective role in response to ischemia. Chemokines such as CCL23 are related to the Th2 autoimmune response and may become important in the repurposing of FDA approved drugs for future therapeutic studies. Variations in proteases, ischemia timeline, patient co-morbidities, reperfusion injury, and basal levels of systemic protein expression may all contribute to the finding of lower intracranial protein expression. Future studies will focus on parsing out the influence of these potential factors as well as on relationships between proteomic changes and patient comorbidities, sex, infarct severity and functional recovery. Proteomic data reported here may provide a scientific springboard for identifying clinically relevant biomarkers, as well as targets for much-needed neuroprotective and neuroreparative pharmacotherapies.

Disclosures B. Maglinger: None. J. Frank: None. C. McLouth: None. A. Trout: None. J. Roberts: None. S. Grupke: None. J. Turchan-Cholewo: None. A. Stowe: 4; C; Cerelux, LLC. J. Fraser: 1; C; AHA, University of Kentucky. 2; C; Stream Biomedical, Medtronic, Penumbra. 4; C; Cerelux, LLC; Fawkes Biotechnology. K. Pennypacker: 4; C; Cerelux, LLC.
$\mathrm{E}-078$

ASSESSMENT OF COMPUTED TOMOGRAPHY PERFUSION SOFTWARE IN MISDIAGNOSIS OF ACUTE ISCHEMIC STROKE PATIENT THROMBECTOMY ELIGIBILITY

${ }^{1} \mathrm{R}$ Rava*, ${ }^{2} \mathrm{M}$ Mokin, ${ }^{3} \mathrm{M}$ Waqas, ${ }^{3} \mathrm{~J}$ Davies, ${ }^{3} \mathrm{E}$ Levy, ${ }^{4} \mathrm{Y}$ Hoi, ${ }^{3} \mathrm{~A}$ Siddiqui, ${ }^{1} \mathrm{C}$ Ionita, ${ }^{3} \mathrm{~K}$ Snyder. ${ }^{1}$ Biomedical Engineering, University at Buffalo, Buffalo, NY; ${ }^{2}$ Neurosurgery, University at Buffalo, Tampa, FL; ${ }^{3}$ Neurosurgery, University at Buffalo, Buffalo, NY; ${ }^{4}$ Canon Medical Systems Inc., Tustin, CA

\subsection{6/neurintsurg-2020-SNIS.112}

Introduction/Purpose Computed tomography perfusion (CTP) is a common imaging modality utilized to assess acute ischemic stroke (AIS) patient eligibility for mechanical thrombectomy. This assessment is done using an ischemic tissue ratio (penumbra to infarct) which is required to be greater than 1 for patients to be eligible for thrombectomy. Since various CTP software utilize different perfusion parameters and thresholds, there are known to be discrepancies in the amount of ischemic tissue quantified for each and therefore conflicting decisions regarding thrombectomy eligibility across software. This study aimed to assess the volumetric agreement of infarct between CTP and 24-hour follow-up fluid-attenuation inversion recovery (FLAIR) magnetic resonance imaging (MRI) along with the number of patients who would be misdiagnosed as ineligible for thrombectomy based on penumbra to infarct ratios by each CTP software.

Materials and Methods Eighty-one emergent large vessel occlusion AIS patients who underwent successful reperfusion (thrombolysis in cerebral infarction $2 b / 2 c / 3$ ) were included in this study. Predicted infarct and penumbra volumes were quantified within RAPID, Sphere, and Vitrea CTP software and compared with infarct measurements from FLAIR MRI. The following CTP parameters and thresholds were used to quantify ischemic tissue through comparison with contralateral hemispheres: RAPID: infarct $=30 \%$ reduction of cerebral blood flow $(\mathrm{CBF})$, penumbra $=6$ second increase in time to reach maximal residue function; Sphere: infarct $=25 \%$ reduction of CBF (and 5 second increase of time-to-peak (TTP)), penumbra $=5$ second increase in TTP; Vitrea: infarct $=38 \%$ reduction in cerebral blood volume (and 5.3 second increase in TTP or $55 \%$ reduction in mean-transit-time), penumbra $=5.3$ second increase in TTP or 5.8 second increase in delay time or $58 \%$ reduction in CBF. Penumbra to infarct ratios were calculated utilizing each software.

Results Mean infarct differences, represented as 95\% confidence intervals, between each software and FLAIR MRI are as follows: $\mathrm{RAPID}=6.96 \pm 4.93 \mathrm{~mL}, \quad$ Sphere $=-0.07 \pm 6.11 \mathrm{~mL}$, Vitrea $=4.13 \pm 4.73 \mathrm{~mL}$. Mean absolute errors for each CTP software compared with FLAIR MRI are: RAPID $=14.48 \mathrm{~mL}$, Sphere $=15.43 \mathrm{~mL}$, Vitrea $=11.44 \mathrm{~mL}$. Mean penumbra to infarct ratios for each CTP software are: $\mathrm{RAPID}=70.93$; Sphere $=32.72$; Vitrea $=12.33$. Total number of patients misdiagnosed as ineligible for thrombectomy (penumbra to infarct ratio less than 1 but successful reperfusion was conducted) for each software: RAPID $=1.23 \%(1 / 81)$, Sphere $=6.17 \% \quad(5 / 81)$, Vitrea $=25.93 \%(21 / 81)$.

Conclusions Sphere provided the most accurate results regarding the closest predicted CTP infarct volume to the true FLAIR MRI infarct volume, although with slightly higher error in its measurements compared to RAPID and Vitrea. Penumbra to infarct ratio analysis indicated RAPID and Sphere to provide the most accurate assessment regarding patient eligibility for thrombectomy. However, it appears sacrificing ischemic tissue measurement precision is required for more accurate inclusion 
of patients as thrombectomy eligible. This is seen by the significantly high mean penumbra to infarct ratio for RAPID which may suggest a caution overestimation of penumbra to label as many patients as possible as thrombectomy eligible for the purpose of regaining lost neurological function.

Disclosures R. Rava: None. M. Mokin: 1; C; NIH grant support R21NS109575. 2; C; Canon Medical Systems Corporation, Cerebrotech, Imperative care. M. Waqas: None. J. Davies: 1; C; National Center for Advancing Translational Sciences of the National Institutes of Health under award number KL2TR001413. 3; C; Penumbra. 4; C; RIST Neurovascular. E. Levy: 2; C; Claret Medical, GLG Consulting, Guidepoint Global, Imperative Care, Medtronic, Rebound, StimMed. 4; C; NeXtGen Biologics, RAPID Medical, Claret Medical, Cognition Medical, Imperative Care (formerly the Stroke Project), Rebound Therapeutics, StimMed, Three Rivers Medical. Y. Hoi: 5; C; Canon Medical Systems Inc. A. Siddiqui: 2; C; Amnis Therapeutics, Boston Scientific, Canon Medical Systems USA Inc, Cerebrotech Medical Systems Inc, Cerenovus, Corindus Inc, Endostream Medical Ltd, Guidepoint Global Consulting, Imperative Care, Integra LifeSciences Corp, Medtronic, MicroVention, Northwest University-DSMB Chair for HEAT Trial, Penumbra, Q'Apel Medical Inc, Rapid Medical, Rebound Therapeutics Corp, Serenity Medical Inc, Silk Road Medical, StimMed, Stryker, Three Rivers Medical, Inc., VasSol, W.L. Gore \& Associates. 4; C; Amnis Therapeutics, Apama Medical, Blink TBI Inc, Buffalo Technology Partners Inc, Cardinal Consultants, Cerebrotech Medical Systems, Inc, Cognition Medical, Endostream Medical Ltd, Imperative Care, International Medical Distribution Partners, Neurovascular Diagnostics Inc, Q'Apel Medical Inc, Rebound Therapeutics Corp, Rist Neurovascular Inc, Serenity Medical Inc, Silk Road Medical, StimMed, Synchron, Three Rivers Medical Inc, Viseon Spine Inc. C. Ionita: 1; C; Equipment grant from Canon Medical Systems, Cummings Foundation support. K. Snyder: 2; C; Canon Medical Systems Corporation, Penumbra Inc, Medtronic, Jacobs Institute.

\section{E-079 PRE-HOSPITAL STROKE TRIAGE DIRECTLY TO THROMBECTOMY CAPABLE CENTERS USING NYC S- LAMS - PRELIMINARY DATA}

${ }^{1} \mathrm{~B}$ Kim*, ${ }^{2} \mathrm{~J}$ Morey, ${ }^{1} \mathrm{~L}$ Stein, ${ }^{3} \mathrm{M}$ Redlener, ${ }^{4} \mathrm{~J}$ Fifi. ${ }^{1}$ Neurology, Icahn School of Medicine at Mount Sinai, New York, NY; ${ }^{2}$ Neurosurgery, Neurology, Icahn School of Medicine at Mount Sinai, New York, NY; ${ }^{3}$ Emergency Medicine, Icahn School of Medicine at Mount Sinai, New York, NY; ${ }^{4}$ Neurosurgery, Icahn School of Medicine at Mount Sinai, New York, NY

10.1136/neurintsurg-2020-SNIS.113

Introduction/Purpose Management of acute ischemic stroke (AIS) in patients with an emergent large vessel occlusion (ELVO) has changed dramatically with endovascular therapy (EVT). Stroke systems of care have evolved to ensure timely EVT in addition to IV thrombolysis (IVT). In collaboration with the Greater New York Hospital Association and American Heart Association, the Fire Department of New York (FDNY) created the first triage protocol in our region to directly route suspected ELVO patients to the nearest thrombectomy capable stroke center (TSC). We sought to describe the results of this triage protocol from initiation in April 2019 to February 2020.

Materials and Methods The FDNY and regional emergency medical advisory committee adapted the Los Angeles Motor Scale (LAMS), with the addition of 'Speech,' to develop a clinical stroke scale for EMS personnel to use in the field: SLAMS. With a S-LAMS score $\geq 4$, EMS contact the main operating center for permission to reroute to the nearest TSC. We conducted a retrospective review of patients triaged to our urban health system using this protocol. The main outcome was the percentage of patients successfully triaged with confirmed ELVO. Time metrics, final diagnosis, National Institute of Health Stroke Scale (NIHSS), and other AIS measures were also analyzed.

Results There were 125 patients (58\% female; median age 71 $\pm 15)$ triaged directly to a TSC. ELVO was confirmed in $32 \%(n=40)$ of patients and 26\% $(n=32)$ underwent EVT. Eight ELVO patients were ineligible for EVT due to either high Modified Rankin Score (mRS) $(n=3)$, infarct evolution $(n=3)$, recanalization after IVT $(n=1)$, or lesion chronicity $(n=1)$. A stroke diagnosis was verified in $75 \%(n=94)$ of triaged patients (71 ischemic and 23 hemorrhagic) regardless of ELVO status. The median S-LAMS score amongst ELVO patients was $6 \pm 1$ (initial provider NIHSS 16 \pm 7 ); score for non-ELVO stroke patients was $5 \pm 1$ (initial provider NIHSS 9 $\pm 7)$. The median hospital arrival to IVT was $41 \pm 36$ minutes $(58 \%[\mathrm{n}=72]$ eligible, $30 \%[\mathrm{n}=37]$ received) and hospital arrival to groin puncture was 1 hour $42 \pm 35$ minutes. The median time from EMS triage to hospital notification was 6 \pm 3 minutes and notification to arrival was $10 \pm 6$ minutes. Occluded vessels included Left M1 $(n=12)$, Left M2 $(n=6)$, Right M1 (n=7), Right M2 (n=3), Left ICA $(n=3)$, R ICA $(n=4)$, and Basilar $(n=1)$ arteries. Top non-stroke diagnoses were seizures $(n=12)$, brain neoplasm $(n=4)$, and transient ischemic attack $(n=2)$.

Conclusion S-LAMS $\geq 4$ correctly identified 32\% ELVO and $75 \%$ stroke patients in this cohort. One quarter of triaged patients received EVT; those excluded mainly had high mRS or established infarct. Relatively short times from triage to notification and arrival, in addition to high yield of correctly triaged ELVO and AIS patients suggest benefit from this triage protocol. Non-stroke patients were diagnosed with conditions that could mimic strokes on presentation. Further analysis is indicated to compare this alternative triage protocol to traditional stroke service delivery models.

Disclosures B. Kim: None. J. Morey: None. L. Stein: None. M. Redlener: None. J. Fifi: None.

\section{E-080 RISK FACTORS OF POST THROMBECTOMY MORTALITY IN ACUTE ANTERIOR CIRCULATION ISCHEMIC STROKE: SINGLE COMPREHENSIVE STROKE CENTER EXPERIENCE}

${ }^{1} \mathrm{~A}$ Toma*, ${ }^{2} \mathrm{~A}$ Vijayashankar, ${ }^{1} \mathrm{~N}$ Haranhalli, ${ }^{2} \mathrm{R}$ Zampolin, ${ }^{1} \mathrm{D}$ Altschul, ${ }^{1} \mathrm{~A}$ Brook, ${ }^{3} \mathrm{~S}$ Lee. ${ }^{1}$ Radiology and Neurosurgery, Albert Einstein College of Medicine, Montefiore Medical Center, Bronx, NY; ${ }^{2}$ Radiology, Albert Einstein College of Medicine, Montefiore Medical Center, Bronx, NY; ${ }^{3}$ Radiology, Neurology and Neurosurgery, Albert Einstein College of Medicine, Montefiore Medical Center, Bronx, NY

\subsection{6/neurintsurg-2020-SNIS. 114}

Background and Purpose Mechanical thrombectomy has significantly improved post-ischemic stroke clinical outcomes. However, the post-ischemic stroke mortality rate appears to be unchanged. We reviewed potential risk factors that can be related to mortality in patients who underwent mechanical thrombectomy.

Materials and Methods A retrospective review was conducted in acute anterior circulation ischemic stroke patients who underwent mechanical thrombectomy but expired within 90 